\title{
Binary Tomography Reconstruction by Particle Aggregation
}

\author{
Mohammad Majid al-Rifaie and Tim Blackwell \\ Department of Computing Goldsmiths, University of London, New Cross, London \\ SE14 6NW, UK, \\ m.majid@gold.ac.uk
}

\begin{abstract}
This paper presents a novel reconstruction algorithm for binary tomography based on the movement of particles. Particle Aggregate Reconstruction Technique (PART) supposes that pixel values are particles, and that the particles can diffuse through the image, sticking together in regions of uniform pixel value known as aggregates. The algorithm is tested on four phantoms of varying sizes and numbers of forward projections and compared to a random search algorithm and to SART, a standard algebraic reconstruction method. PART, in this small study, is shown to be capable of zero error reconstruction and compares favourably with SART and random search.
\end{abstract}

Keywords: Binary tomography, Discrete tomography, Particle Aggregation, underdetermined linear systems

\section{Introduction}

Tomographic reconstruction is the process of inferring the internal structure of an object from a set of projected images. The projected images are records of the quantity of penetrating radiation that has passed through, or has been emitted from the interior of, the object in question. There are many applications, ranging from medical imaging (CT, SPECT, PET and MRI) $[3,4,16]$ to oceanography (seismic tomography) [14] and quantum tomography (quantum state tomography) [5].

Although an exact reconstruction is possible by use of the inverse Radon transform, in practice the discrete nature of the imaging, and the finite number of available projections, mean that approximate, discrete, techniques must be employed. The continuous density distribution of the object is modelled as a grid of pixels and the projections are acquired in bins becasue cameras consist of arrays of detectors of finite size [4].

Even after discrete modelling, the remaining mathematical problem may be ill-defined due to underdetermination: the number of independent relationships amongst the unknown quantities is fewer than their number. As a result, the solution of the inverse problem is not unique, and indeed very many solutions might exist. 
This incompleteness of data arises from cost, time and geometrical concerns. For instance, the importance of cost reduction in industrial applications results in shortened scan duration and fewer projected images; similarly, in electron tomography, the damage caused to the sample by the electron beam reduces the number of collectable projections [13].

The classical filtered back projection $[11,7]$ technique is a relatively quick and effective reconstruction procedure. However, increasing computation power means that algebraic reconstruction techniques (algebraic-RT or ART) are gaining eminence. This is due to ART's potential for greater accuracy, albeit at increased time of execution.

The first ART algorithm was a rediscovery [6] of the Kaczmarz method for solving linear equations [10]. An improved Kaczmarz method for image reconstruction, SART, (simultaneous-ART) was proposed by Andersen and Kac [1]. SART remains popular to this day and has been the subject of mathematical analysis (for example, [9]).

Prior knowledge can inform algorithms and speed up computation. For example, if it is known that the object is composed of just a few regions of homogenous density, discrete tomography can be employed. The aim is to reconstruct an image that is composed of just a few greyscale values. And, as an extreme instance of discrete tomography, if just two greyscale values are assumed, corresponding to the interior and exterior of the object, the problem is to find a binary reconstruction [8].

The aim of this paper is to investigate a new binary reconstruction technique based on the aggregation of particles. The idea is to suppose that pixel values 0 and 1 represent particles that may be absent or present in a particular cell (a pixel), and for isolated particles to move freely until they meet, and thereupon stick to, clusters of other particles. The underlying assumption is that the preferred solutions to the inverse problem will be those solutions that are more homogeneous.

The paper continues with an overview of tomography and of reconstruction. Then, the aggregation algorithm, Particle aggregate-RT (PART) is specified; a section detailing a sequence of experiments that tests and compares PART to SART and to a random search on a number of phantoms (i.e. pre-prepared exact images) follows. The results are then reported and evaluated. The paper ends with a summary of the main findings and suggestions for future research.

\section{Tomography and algebraic reconstruction}

There are two important imaging modalities, parallel beam and fan beam tomography. In either modality, an array of detectors is rotated to lie at a number of (usually) equally spaced angles in $[0, \pi)$. Fig. 1 shows the two modalities and the pixellated representation of the object. Ideally, if the detectors have perfect collimators, each detector will record the amount of radiation received in a finite width beam. 
Fig. 1: Tomography geometry

Left: parallel beam geometry; right: fan beam geometry
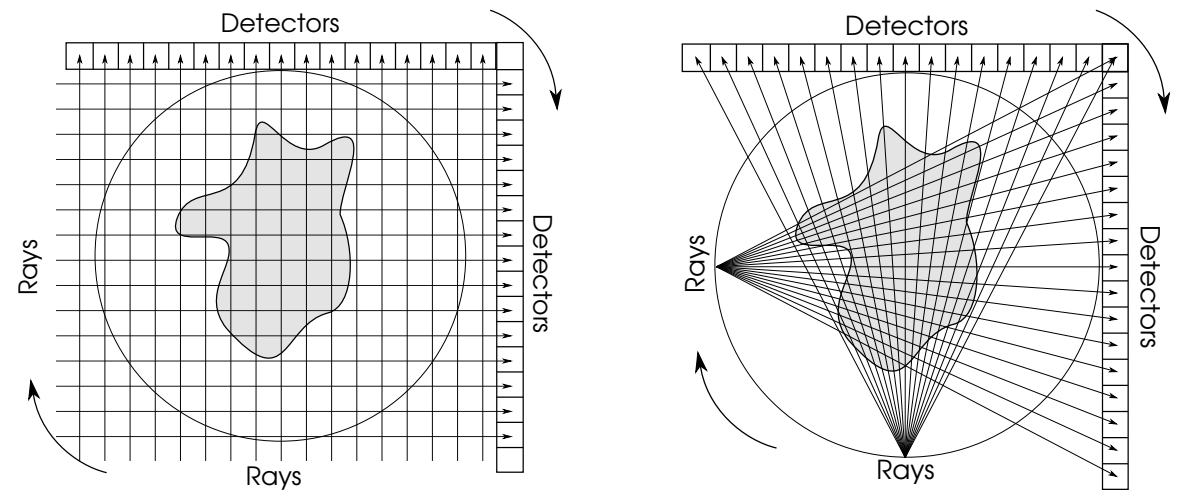

However, an approximate model of the physical measurement must be built in order to formalise the mathematical reconstruction problem. This approximation is called the forward model. Beams are typically modelled by parallel rays (Fig. 1 left). Each ray is incident on the centre of each detector or projection bin. The imaging process is approximated by a projection matrix $A \in \mathbb{R}_{\geq 0}^{m \times n}$ where $m$ is the total number of rays collected (equal to the number of rays at each projection angle multiplied by the number of projection angles) and $n$ is the number of pixels in the reconstructed image. If $b \in \mathbb{R}^{m}$ is a vector of detector values, the continuous/discrete reconstruction problem can be stated as:

$$
\text { find } x\left\{\begin{array}{l}
\in \mathbb{R}^{n} \\
\in\{0,1, \ldots, k-1\}^{n}, k>1
\end{array} \quad \text { such that } A x=b\right. \text {. }
$$

The binary problem is $k=2$ i.e. with $x \in\{0,1\}^{n}$.

The methods used to compute the entries $A_{i j}$ of the projection matrix vary. A simple procedure is to count the number of pixels that each ray passes through. The more refined line model uses the length of the intersection between the ray and the pixel.

Since the equation $A x=b$ is, in general, underdetermined, it cannot be inverted. Instead an approximate solution $y$ must be obtained (for example, by filtered back projection, or SART). This trial solution is forward projected according to the measurement model:

$$
A y=c
$$

with an associated $l_{p}$ error

$$
\epsilon(y)=\|b-c\|_{p}
$$


where the $l_{p}, p \geq 1$, norm is defined

$$
\|v\|_{p} \equiv\left(\sum|x|^{p}\right)^{\frac{1}{p}}
$$

An iterative scheme will produce a sequence of candidate solutions, $y^{(k)}, k=$ $1,2, \ldots$, of decreasing error.

\section{Reconstruction by Particle Aggregation}

In many applications, the reconstructed image is expected to consist of patches of various sizes of uniform pixel value, since many physical objects of interest consist of uniform structures. Non-uniform regions with randomly varying pixel value would be construed as noisy and unphysical. Relevant reconstructed images are therefore those with low entropy.

This observation suggests the following assumption: given a number of distinct candidate reconstructions, $\{y: A y=c\}$, with identical error $\epsilon(y)$, the preferred reconstruction is the one with the lowest entropy (or one of the reconstructions of lowest entropy, in the case of non-uniqueness). It would clearly be beneficial to equip a reconstruction algorithm with this assumption, under those conditions where the assumption might be expected to hold.

The principle idea underlying the aggregation technique proposed in this paper and motivated by the low-entropy assumption, is to suppose that pixel values are mobile particles, moving from pixel to pixel. The low-entropy assumption is implemented by requiring that particles stick together in clusters or aggregates uniform pixel value.

A model of aggregation for any random deposition process that is dominated by diffusive transport, for example electodeposition and mineral growth, was proposed in 1981 by Witten and Sander [18]. Their model, known as Diffusion Limited Aggregation or DLA, is remarkably simple: a particle is released from a random point on a boundary and subsequently follows a random walk until it strikes a stationary particle at some location within the enclosing boundary. The walking particle sticks to the stationary particle and another particle is released. Surprisingly complex dendrite-like clusters with fractal structure are formed by repeated application of this simple rule.

The reconstruction problem is converted into particle aggregation with the following correspondence:

- image $x \rightarrow$ configuration of particles,

- pixels $\rightarrow$ cells

- pixel values $1 / 0 \rightarrow$ presence/absence of a single particle,

- image $\rightarrow$ a grid of cells.

Furthermore, an objective function

- error function $\rightarrow$ objective function 
converts the growth model into an optimisation problem: only those aggregates that lower the objective function are permitted to form.

A direct implementation of DLA as a reconstructive process would be very expensive since a randomly walking particle might pass by many isolated cells before arriving at a boundary cell; diffusion can be accelerated by causing a particle to jump from cell $a$ to a vacant cell $b$, picked uniformly at random from all vacant cells. Although a jump has been made, the particle might not necessarily 'stick'.

Suppose a particle has jumped from $a$ to $b$ and that $b$ is a boundary cell of a particle cluster. (Note that the boundary might lie within the cluster i.e. bounding a hole). We might suppose that whether the particle sticks or not to the cluster is conditional on the number of occupied neighbours of the boundary cell $b$ relative to neighbour count for cell $a$, and on the fitness of the new configuration. There are a number of ways to deal with a particle that has jumped to a vacant cell but does not stick. For example, it could simply return to $a$. These modifications should ensure that particle diffusion builds aggregates which lower overall entropy and image error.

With these considerations in mind, the Particle Aggregate Reconstruction Technique (PART) can be specified ${ }^{1}$. Algorithm 1 specifies an application of PART to a single particle. Here, $y$ is the reconstructed image, SELECT returns pixels $a, b \in y, a \neq b$, such that $a$ is occupied and $b$ is empty. $n$ is the number of occupied cells in the neighbourhood (Moore or von Neumann) of a particular cell and $\epsilon(a \rightarrow b)$ is the error of the new image with the pixel $a$ set to zero and pixel $b$ set to $1 . u$ is a sample drawn from $U(0,1)$ (the uniform distribution on $[0,1])$.

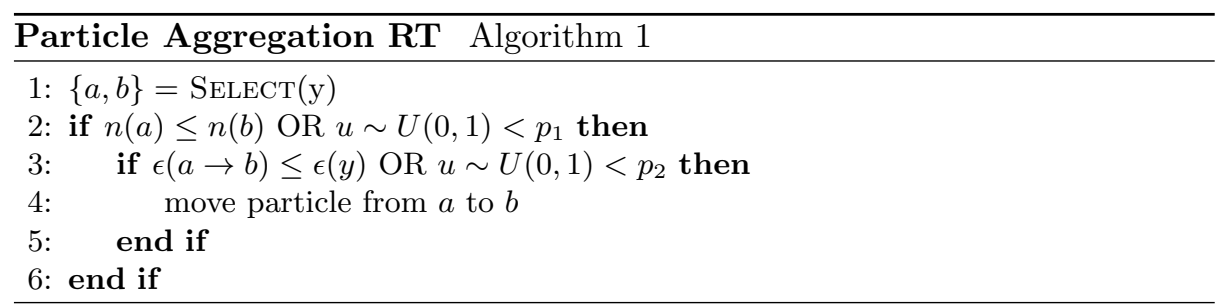

The algorithm has two parameters $p_{1}$ and $p_{2} . p_{1}$ governs the influence of the local neighbourhood constraint: the requirement to move to a neighbourhood of higher local particle density. $p_{1}=1$ corresponds to a random search and the neighbourhood constraint is ignored. A move $a \rightarrow b$ will always be attempted even if the neighbourhood function $n$ is lowered.

In contrast, $p_{2}$ governs the influence of the global constraint on the particle configuration as a whole. If $p_{2}=0$, a move $a \rightarrow b$ will always be rejected if it

\footnotetext{
1 The source code for PART algorithm can be downloaded from http://doc.gold.ac.uk/ map01mm/PART/.
} 
does not lower or equal the current error. The algorithm is greedy. If $p_{2}>0$, the algorithm is not greedy and a configuration with higher error will be accepted with probability $p_{2}$. Movement away from a local minima of $\epsilon$ can occur. In principle, $p_{2}$ might depend on the change in error (and on a steadily reducing temperature parameter as in simulated annealing).

Algorithm 1 specifies a trial update of a single particle. Each application incurs a cost of a single function evaluation $(\epsilon(y))$. The algorithm is iterated until zero error or until a set number of function evaluations (FEs) has been achieved.

As stated by Reynolds [15], the three simple rules of interaction in flocks or swarms are collision avoidance, velocity matching and flock centring. The aggregating particles can be considered as individuals in a swarm. The dynamic rules of particles swarms are of the form:

1. If too close or colliding to neighbouring particles, move away

2. Else if too far from neighbours, move closer.

where rule 1 opposes crowding and rule 2 brings the particles together in a swarm. The single occupancy condition implements the anti-crowding rule, and the (conditional) move to a neighbourhood of higher particle density, as measured by the neighbourhood function $n$ implements rule 2 . The error function $\epsilon(y)$ imposes a global constraint on the swarm as a whole.

\section{Experiments and results}

This section presents a series of experiments to investigate the performance of PART in binary image reconstruction. Three experiments were designed. The first, and preliminary, experiment, aims at finding a suitable value for the local constraint parameter $p_{1}$ for a single phantom of one size only. The second experiment investigates the convergence properties of PART and random search, which can be seen as a limiting case of PART. The final experiment provides a comparison between random search, the commonly used reconstruction algorithm, Simultaneous Algebraic Reconstruction Technique (SART), and PART with $p_{1}$ set to the empirical value determined in the first experiment.

\subsection{Methodology}

Forward model The acquisition geometry used for the experiments is parallel beam topology and the experiments use simulated objects (i.e. virtual phantoms). In all cases, the elements of the projection matrix were calculated from the line model.

Phantoms Phantoms 1 and 2 (see Fig. 2) are commonly used in binary tomography [17] and the third and fourth phantoms resemble the Jaszczak phantoms used to calibrate the SPECT and PET scanning machines. The size of all the 
phantoms is $512 \times 512$. To carry out the experiments in images with different sizes, the phantoms or reference images have been scaled to create images of varying sizes (namely, $32 \times 32,64 \times 64$ and $128 \times 128$ ).

PART PART is used with Moore neighbourhood. There are a number of alternatives for line 1 of Algorithm 1, the selection step in PART. The purpose of this step is to find an occupied cell, $a$, and a vacant cell, $b$. The following experiments use random selection: $a$ and $b$ are selected uniformly at random from the sets of all occupied/unoccupied cells. A list implementation would have been efficient, but since the numbers of occupied/unoccupied cells is roughly similar, uniform sampling over the entire grid $y$ was used due to the ease of implementation and small time overhead. Algorithm 2 specifies SELECT; $U(y)$ is a uniform random selection of a single cell from the grid $y$. The value of the global constraint parameter $p_{2}$ was fixed, in all experiments, to zero.

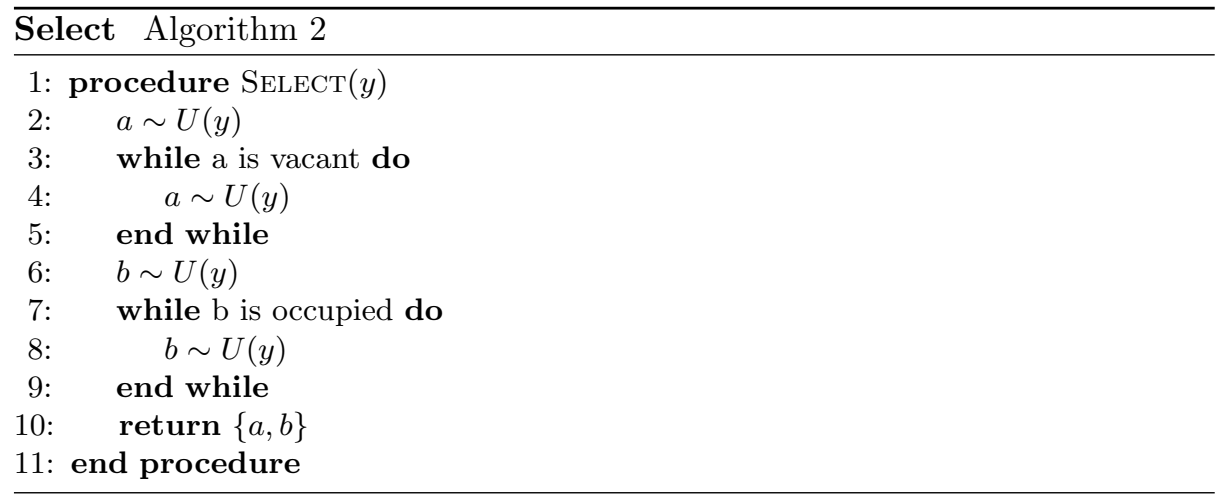

Random Search (RS) For the purposes of these experiments, random search is defined as the PART algorithm with the neighbourhood parameter $p_{1}$ set

Fig. 2: Phantom images used in the experiments

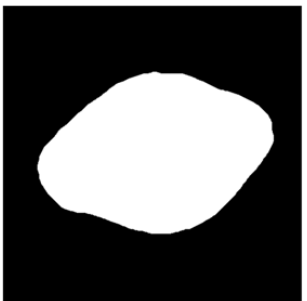

(a) Phantom 1

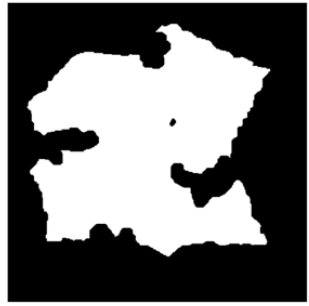

(b) Phantom 2

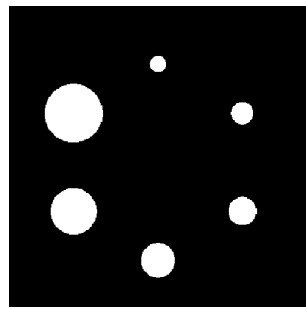

(c) Phantom 3

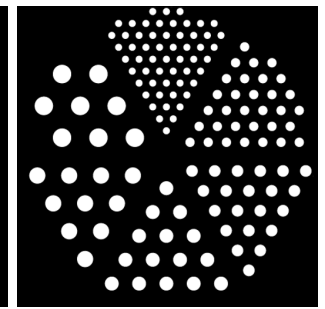

(d) Phantom 4 
to 1 with the consequence that a particle will always attempt a move to an unoccupied cell $b$ even if the neighbour count of $b, n(b)$, is less than $n(a)$.

SART The implementation of SART used here was based on Andersen and Kac's algorithm, [1]. The projection angles were selected uniformly at random $([2])$. The value of the relaxation parameter $\lambda$ was set to 1.9 in accordance with the recommendation of [12].

SART needs to be modified for binary reconstruction since in the unaltered form SART produces a continuum of pixel values. The reconstructed image also needs to be normalised in order to make error comparisons. The following modifications were made: any negative pixel values occurring after updating at any angle were set to zero; the final image $y$ after updating all projection angles was normalised so that the total pixel value count of the phantom image and the reconstructed image were equal; $y$ was thresholded at the average pixel value so that values below the average were set to zero, values above or equal tot he average were set to 1 .

Measure The principle performance measure is the $l_{1}$ norm, $\epsilon(y)=\left(\sum|b-c|\right)$ where, for the phantom image $x, A x=b$ and for the reconstructed image, $A y=c$. A secondary measure, used for comparison amongst iterated algorithms, is the number of evaluations of $\epsilon$ (function evaluations or FEs) needed to attain a given error.

\subsection{Experiment I: neighbourhood constraint parameter, $p_{1}$}

The aim of this preliminary experiment is to find a suitable value for the parameter $p_{1}$ which determines the probability of ignoring the neighbourhood constraints. Phantom 3 is used in this experiment (see Fig. 2c), with dimension $32 \times 32$ and 32 angles of projections. The graphs in Fig. 3 show the result of a typical PART run. The termination condition is 50,000 FEs.

As shown in Fig. 3(b), $p_{1}=\{0.1,0.2,0.3\}$ produces zero error, and when the number of FEs needed to achieve this error is taken into account (Fig. 3(a)), the most suitable value is $p_{1}=0.1$.

\subsection{Experiment II: PART and RS convergence}

Fig. 4 reports on a typical run of PART at $p_{1}=0.1, p_{2}=0$ and $\mathrm{RS}\left(p_{1}=1, p_{2}=\right.$ $0)$ on phantoms of size $64 \times 64$ with 32 angles $(64 \times 64 \times 32)$. The termination condition is zero error or 50,000 FEs.

As shown in the graphs in Fig. 4, PART outperforms RS before the maximum allowed iterations for phantoms 1-3. RS appears to be superior for phantom 4 for large numbers of FE's (beyond 30000). This is possibly due to the presence of isolated pixels with value 1 in the phantoms as a result of scaling down the image to $64 \times 64$; PART will struggle to place a particle on an isolated $(n(b)=0)$ cell. 
Fig. 3: Error and function evaluations (FEs) with varying $p_{1}$ values

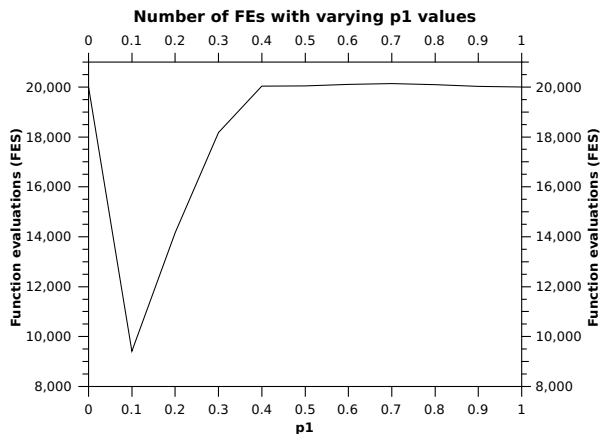

(a) FEs vs $p_{1}$

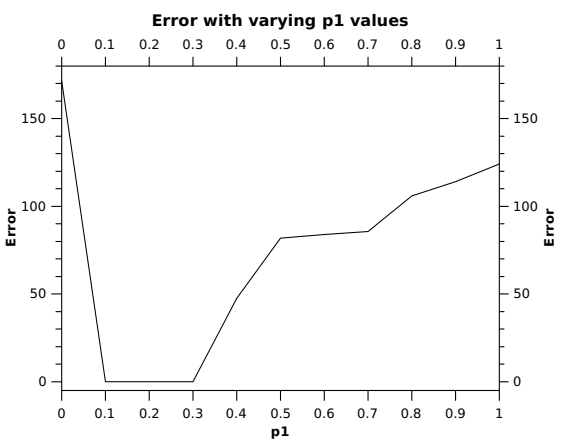

(b) Error vs $p_{1}$

The PART convergence curves begin to level off at about 10000 particle updates, i.e. at about $8 \%$ of the number of measurements $\left(64^{2} \times 32\right)$.

Fig. 4: Convergence plots of PART and RS algorithms
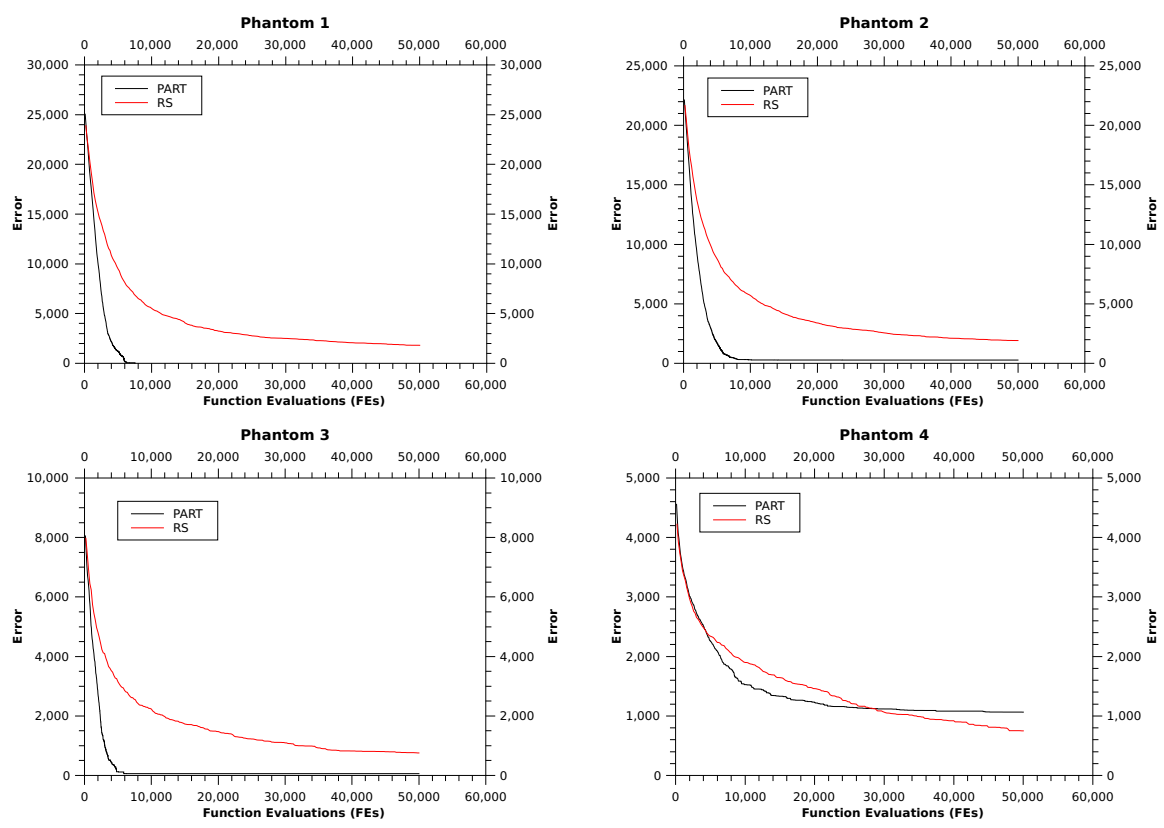


\subsection{Experiment III: PART, RS and SART comparisons}

The aim of this experiment is to compare PART, RS and SART reconstructions of the four phantoms for various sizes and numbers of projection angles. 30 runs were conducted for each phantom and for the each algorithm in order to acquire adequate statistics. The termination condition for each run is zero error or 50,000 FEs. (For the purposes of this study, the number of FEs does not vary with the number of measurements.)

Figs. 5 and 6 (and Table 2) illustrate the performance of the three algorithm in two separate sets of experiments, using phantoms of size $32 \times 32$ with 16 projections $(32 \times 32 \times 16)$ and $64 \times 64$ with 32 projections $(64 \times 64 \times 32)$ respectively. As shown in the figures, increasing the size of the phantoms, PART distances itself from RS and SART in terms of the error value and outperforms both algorithms (with the exception of phantom 4 for the reason stated before).

Fig. 5: Error values in PART, RA and SART $(32 \times 32 \times 16)$
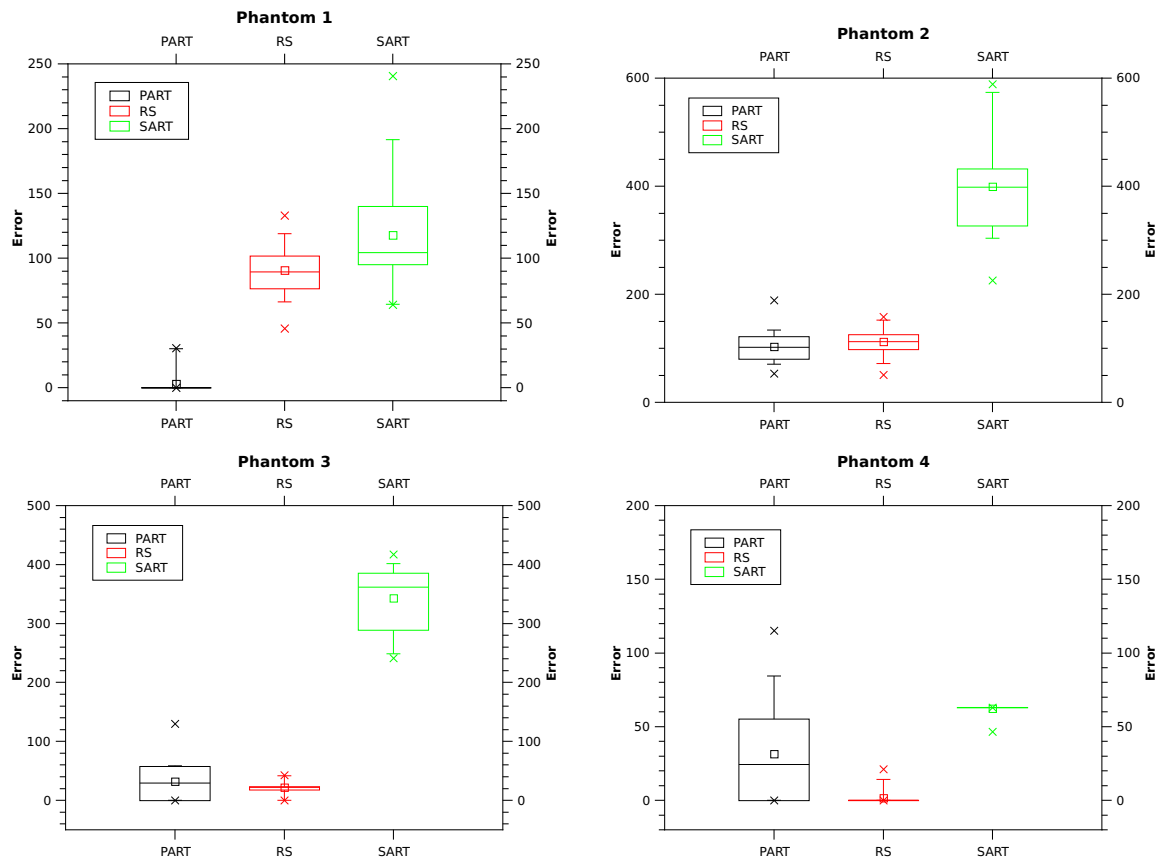

In order to conduct the statistical analysis and identify the presence of any significant difference in the performance of the algorithms, Wilcoxon $1 \times 1$ nonparametric statistical test is deployed. Table 1 confirms the findings reported and shows that in the experiments for $64 \times 64 \times 32$, PART significantly outperforms RS in all cases (except in phantom 4); additionally it is shown that PART 
Fig. 6: Error values in PART, RA and SART $(64 \times 64 \times 32)$
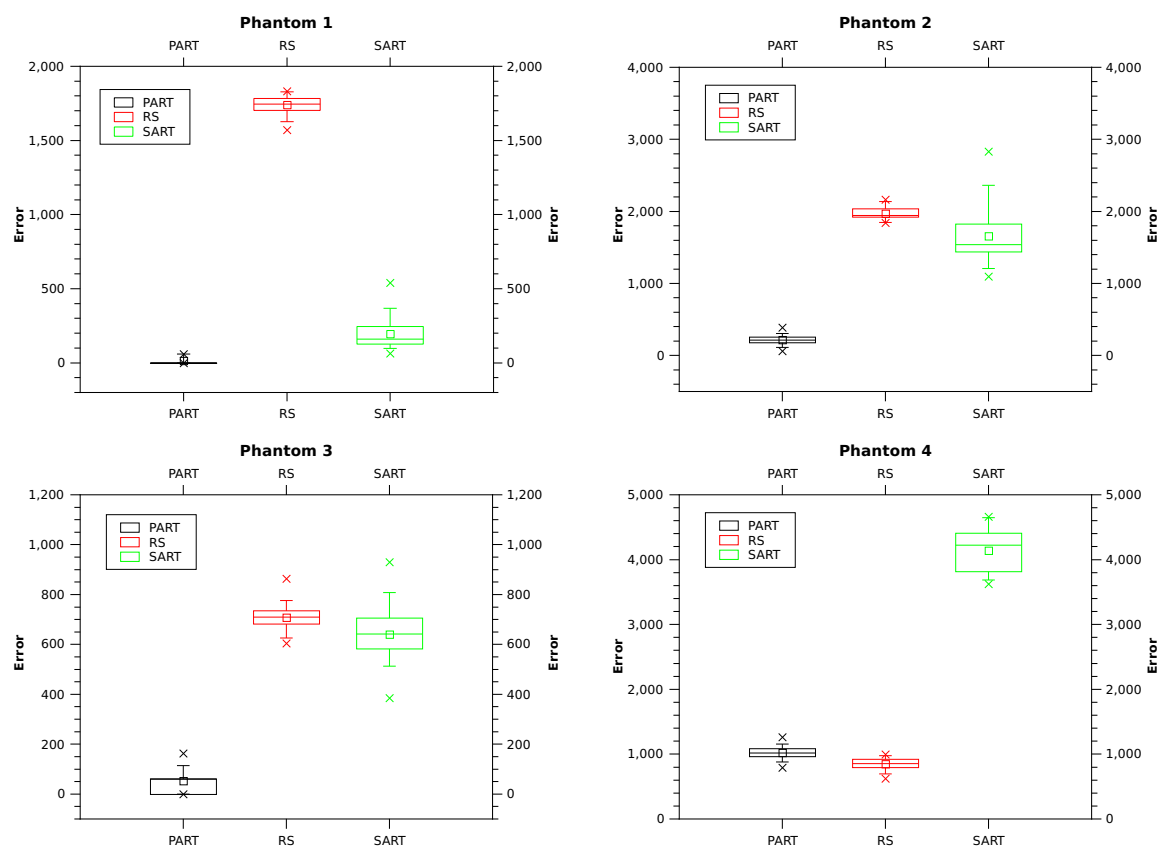

significantly outperforms SART in all cases in both $32 \times 32 \times 16$ and $64 \times 64 \times 32$ experiments. While RS outperforms SART in all cases in $32 \times 32 \times 16$ experiments, with scaling the image to $64 \times 64$ the results are reversed. Phantom 4 remains the only case where RS significantly outperforms both PART and SART algorithms. 
Table 1: Statistical analysis of the performance of the algorithms Based on Wilcoxon $1 \times 1$ Non-Parametric Statistical Test, if the error difference between each pair of algorithms is significant at the $5 \%$ level, the pairs are marked. $\mathrm{X}-\mathrm{o}$ shows that the left algorithm is significantly outperforming its counterpart algorithm; and $\mathrm{o}-\mathrm{X}$ shows that the right algorithm is significantly better than the one on the left. The figures, $\mathrm{n}-\mathrm{m}$, in the last row present a count of the number of X's and o's in the respective columns.

(a) $32 \times 32 \times 16$

\begin{tabular}{|c|c|c|c|}
\hline & PART - RS & RS - SART & PART - SART \\
\hline Phantom 1 & $\mathrm{X}-\mathrm{o}$ & $\mathrm{X}-\mathrm{o}$ & $\mathrm{X}-\mathrm{O}$ \\
\hline Phantom 2 & - & $X-o$ & $X-o$ \\
\hline Phantom 3 & - & $X-o$ & $\mathrm{X}-\mathrm{o}$ \\
\hline Phantom 4 & $\mathrm{o}-\mathrm{X}$ & $\mathrm{X}-\mathrm{o}$ & $\mathrm{X}-\mathrm{o}$ \\
\hline$\sum$ & $1-1$ & $4-0$ & $4-0$ \\
\hline \multicolumn{4}{|c|}{ (b) $64 \times 64 \times 32$} \\
\hline & PART - RS & RS - SART & PART - SART \\
\hline Phantom 1 & $\mathrm{X}-\mathrm{o}$ & $\mathrm{o}-\mathrm{X}$ & $\mathrm{X}-\mathrm{o}$ \\
\hline Phantom 2 & $\mathrm{X}-\mathrm{o}$ & $\mathrm{o}-\mathrm{X}$ & $\mathrm{X}-\mathrm{o}$ \\
\hline Phantom 3 & $\mathrm{X}-\mathrm{o}$ & $\mathrm{o}-\mathrm{X}$ & $\mathrm{X}-\mathrm{o}$ \\
\hline Phantom 4 & $\mathrm{o}-\mathrm{X}$ & $\mathrm{X}-\mathrm{o}$ & $\mathrm{X}-\mathrm{o}$ \\
\hline$\sum$ & $3-1$ & $1-3$ & $4-0$ \\
\hline
\end{tabular}


Table 2: Comparing PART, RS and SART in $32 \times 32 \times 16$ and $64 \times 64 \times 32$

(a) $32 \times 32 \times 16$

\begin{tabular}{lcccccc}
\hline & & Min & Max & Median & Mean & StDev \\
\hline \multirow{3}{*}{ Phantom 1 } & PART & 0.00 & 30.56 & 0.00 & 3.02 & 9.23 \\
& RS & 45.95 & 133.03 & 89.52 & 90.75 & 18.77 \\
& SART & 64.29 & 240.69 & 104.42 & 118.00 & 44.02 \\
\hline \multirow{2}{*}{ Phantom 2 } & PART & 53.17 & 188.90 & 101.89 & 102.87 & 27.79 \\
& RS & 50.83 & 158.18 & 112.56 & 112.07 & 24.47 \\
& SART & 225.69 & 589.04 & 398.36 & 399.45 & 88.04 \\
\hline \multirow{2}{*}{ Phantom 3 } & PART & 0.00 & 129.82 & 29.06 & 32.15 & 31.06 \\
& RS & 0.00 & 42.60 & 22.03 & 21.79 & 11.58 \\
& SART & 241.41 & 417.06 & 361.53 & 343.15 & 54.09 \\
\hline \multirow{2}{*}{ Phantom 4 } & PART & 0.00 & 115.11 & 24.43 & 31.55 & 33.02 \\
& RS & 0.00 & 21.07 & 0.00 & 1.65 & 5.13 \\
& SART & 46.63 & 62.98 & 62.98 & 62.44 & 2.98 \\
\hline
\end{tabular}

(b) $64 \times 64 \times 32$

\begin{tabular}{lcccccc}
\hline & & Min & Max & Median & Mean & StDev \\
\hline \multirow{2}{*}{ Phantom 1 } & PART & 0.00 & 59.59 & 0.00 & 13.90 & 25.63 \\
& RS & 1571.18 & 1831.95 & 1745.67 & 1740.33 & 68.06 \\
& SART & 63.41 & 539.67 & 160.31 & 194.65 & 102.15 \\
\hline \multirow{2}{*}{ Phantom 2 } & PART & 61.88 & 386.65 & 212.50 & 215.76 & 63.26 \\
& RS & 1841.01 & 2164.48 & 1943.93 & 1972.55 & 85.57 \\
& SART & 1096.49 & 2831.89 & 1537.65 & 1659.28 & 386.71 \\
\hline \multirow{2}{*}{ Phantom 3 } & PART & 0.00 & 163.67 & 59.62 & 52.45 & 42.64 \\
& RS & 604.34 & 864.29 & 709.08 & 708.47 & 53.00 \\
& SART & 384.86 & 929.52 & 641.62 & 640.78 & 106.97 \\
Phantom 4 & PART & 794.81 & 1262.07 & 1017.73 & 1021.08 & 93.57 \\
& RS & 623.06 & 995.50 & 854.67 & 849.62 & 88.21 \\
& SART & 3626.21 & 4663.74 & 4222.92 & 4142.60 & 325.57 \\
\hline
\end{tabular}

In the previous sections of the paper, the success of RS over PART and SART was cautiously attributed to the presence of isolated pixels of value 1 throughout phantom 4 due to scaling down the original phantom.

To put this hypothesis to test, another experiment is designed which uses a larger phantom size of $128 \times 128$ with 16 number of angles in order to evaluate the performance of the algorithms. The results of this experiment is shown in Fig. 
7 and Table 3. The results show, that while RS still outperforms SART, PART which is a simple neighbourhood aware algorithm, exhibits the best performance over all the 30 trials with statistically significant difference (see Table 4).

Fig. 7: Error values in reconstructing phantom 4 using PART, RA and SART in $128 \times 128 \times 16$

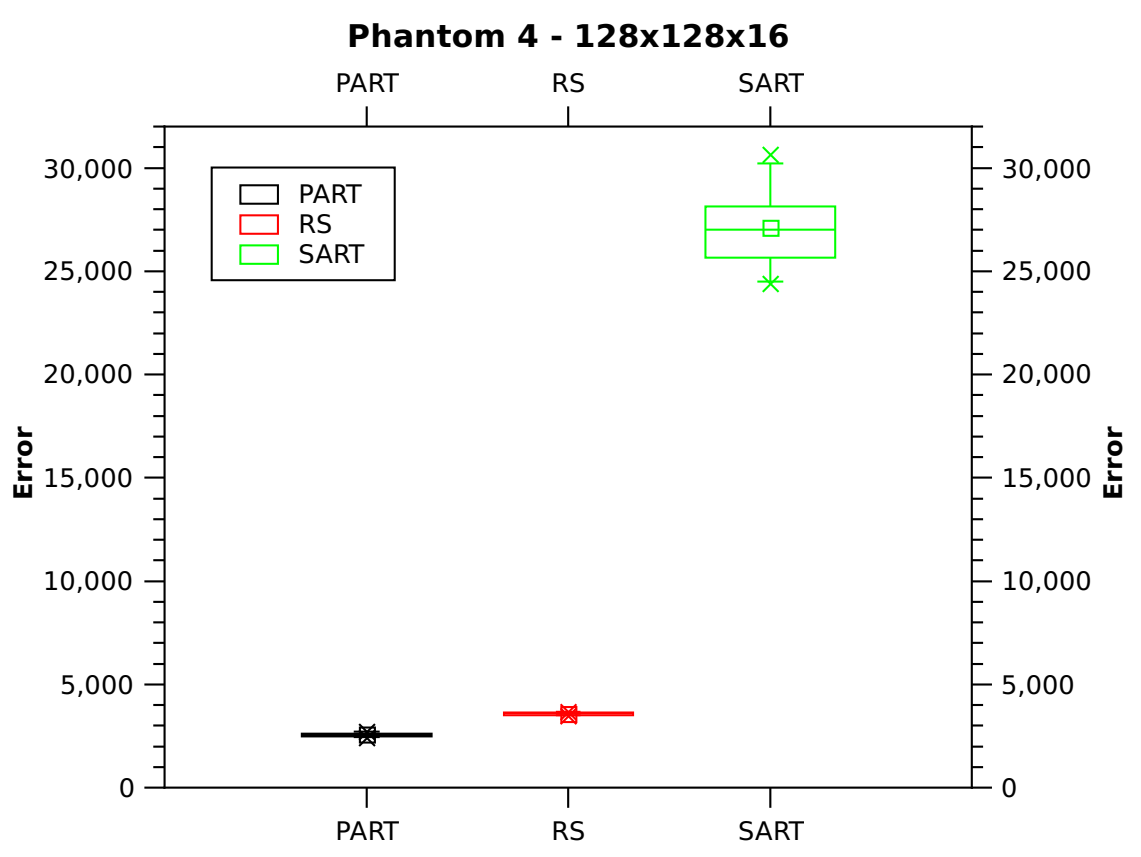

Table 3: Reconstructing phantom 4 using PART, RS and SART in $128 \times 128 \times 16$

\begin{tabular}{lcccccc}
\hline & & Min & Max & Median & Mean & StDev \\
\hline \multirow{3}{*}{ Phantom 4 } & PART & 2398.79 & 2719.48 & 2555.81 & 2564.35 & 84.00 \\
& RS & 3469.15 & 3677.52 & 3589.69 & 3584.50 & 58.11 \\
& SART & 24387.04 & 30642.35 & 27009.73 & 27110.61 & 1867.59 \\
\hline
\end{tabular}


Table 4: Statistical analysis of the performance of the algorithms on phantom 4 in $128 \times 128 \times 16$

\begin{tabular}{cccc}
\hline \multicolumn{4}{c}{$128 \times 128 \times 16$} \\
\hline PART - RS & RS - SART & PART - SART \\
\hline Phantom 4 & $\mathrm{X}-\mathrm{o}$ & $\mathrm{o}-\mathrm{X}$ & $\mathrm{X}-\mathrm{o}$ \\
\hline$\sum$ & $1-0$ & $0-1$ & $1-0$ \\
\hline
\end{tabular}

\section{Conclusions}

This paper has proposed a novel method of binary tomographic reconstruction. PART - particle aggregate reconstruction technique - is based on the idea that an image can be interpreted as a grid of cells populated by particles. Pixel values represent cell occupancy; particles are mobile and diffuse throughout the grid by random jumps, preferably landing adjacent to regions of increased particle density. The algorithm has two free parameters, a neighbourhood constraint probability, $p_{1}$, that controls the preference for jumping to increased density locations, and a global constraint $p_{2}$ that determines how likely the particle is likely to remain at a target cell if the reconstructed image error were to increase by the jump in question.

A series of experiments on four phantoms ranging in size from $32 \times 32$ to $128 \times 128$ suggest that, for $p_{2}=0$ (i.e. no uphill motion in the fitness landscape is allowed), and in the context of the specific trials conducted in this study:

- $p_{1}$ should be set to 0.1 . This means that $10 \%$ of jumps are to locations of decreased neighbourhood particle density.

- PART converges rapidly when compared to random search for phantoms with all nonzero pixel values occuring in connected regions. And in the case that there are isolated nonzero values pixels, PART will find better reconstructions at fewer iterations.

- PART performs (statistically) significantly well when compared to random search and a standard algebraic reconstruction technique for $32 \times 32$ and $64 \times 64$ phantoms, except for the case of isolated nonzero pixel values;

- however for a larger $(128 \times 128)$ phantom with proportionally fewer angles of projection, PART wins out over random search and SART

The dominance of PART - or as its limiting case - random particle diffusion over a standard algebraic technique is suggestive. The algorithm is intuitive, and easily implemented.

However the findings listed above must be tempered with a few provisos. The technique requires many iterations (although the required number is less than the number of linear equations) and it remains to be seen if a more efficient selection mechanism for jumping can provide tolerable run times for large images. The $\left(p_{1}, p_{2}\right) \in[0,1]^{2}$ parameter plane has not been explored and it is expected that 
some fitness landscape climbing would improve performance. The test phantoms used in this study are of limited size and diversity; larger and physically realistic phantoms and object images should be examined. Binary-PART would benefit from a systematic study of the parameters over a wide range of conditions.

This paper has considered binary tomography and the question whether aggregation by particle diffusion can be extended to the general discrete case, is the topic of an ongoing research.

\section{References}

1. Andersen, A., A, K.: Simultaneous algebraic reconstruction technique (sart): a superior implementation of the art algorithm. Ultrason. Img. 6, $81-94$ (1984)

2. Batenburg, K., Sijbers, J.: Dart: A practical reconstruction algorithm for discrete tomography. Image Processing, IEEE Transactions on 20(9), 2542-2553 (Sept 2011)

3. Block, K.T., Uecker, M., Frahm, J.: Undersampled radial mri with multiple coils. iterative image reconstruction using a total variation constraint. Magnetic resonance in medicine 57(6), 1086-1098 (2007)

4. Bruyant, P.P.: Analytic and iterative reconstruction algorithms in spect. Journal of Nuclear Medicine 43(10), 1343-1358 (2002)

5. D'Ariano, G., Presti, P.L.: Quantum tomography for measuring experimentally the matrix elements of an arbitrary quantum operation. Physical review letters 86(19), 4195 (2001)

6. Gordon, R., Bender, R., Herman, G.T.: Algebraic reconstruction techniques (art) for three-dimensional electron microscopy and x-ray photography. Journal of Theoretical Biology 29(3), 471 - 481 (1970), http://www.sciencedirect.com/science/article/pii/0022519370901098

7. Herman, G.T.: Fundamentals of computerized tomography: image reconstruction from projections. Springer Science \& Business Media (2009)

8. Herman, G.T., Kuba, A.: Advances in discrete tomography and its applications. Springer Science \& Business Media (2008)

9. Jiang, M., Wang, G.: Convergence of the simultaneous algebraic reconstruction technique (sart). In: Signals, Systems and Computers, 2001. Conference Record of the Thirty-Fifth Asilomar Conference on. vol. 1, pp. 360-364 vol.1 (Nov 2001)

10. Kaczmarz, S.: Angenaherte auflosung von systemen linearer gleichungen. Bull. Internat. Acad. Polon.Sci. Lettres A p. 335357 (1937)

11. Kak, A.C., Slaney, M.: Principles of computerized tomographic imaging. Society for Industrial and Applied Mathematics (2001)

12. Kazemini, E., Nedialkov, N.: An empirical study of algebraic reconstruction techniques. In: J., T., N., J. (eds.) Computational vision and Medical Image Processing IV, pp. 93 - 98. Taylor and Francis (2014)

13. Midgley, P.A., Dunin-Borkowski, R.E.: Electron tomography and holography in materials science. Nature materials 8(4), 271-280 (2009)

14. Nolet, G.: Seismic wave propagation and seismic tomography. In: Seismic tomography, pp. 1-23. Springer (1987)

15. Reynolds, C.W.: Flocks, herds and schools: A distributed behavioral model. In: ACM Siggraph Computer Graphics. vol. 21, pp. 25-34. ACM (1987)

16. Ter-Pogossian, M.M.: Positron emission tomography (pet). In: Diagnostic Imaging in Medicine, pp. 273-277. Springer (1983) 
17. Van Dalen, B.: Stability results for uniquely determined sets from two directions in discrete tomography. Discrete Mathematics 309(12), 3905-3916 (2009)

18. Witten, T.A., Sander, L.M.: Diffusion-limited aggregation, a kinetic critical phenomenon. Phys. Rev. Lett. 47, 1400-1403 (Nov 1981), http://link.aps.org/doi/10.1103/PhysRevLett.47.1400 